\title{
THE THERMOELASTIC PROBLEM FOR A PENNY-SHAPED ANTICRACK WITH HEAT CONDUCTIVITY IN A TRANSVERSELY ISOTROPIC SPACE
}

\author{
ANDRZEJ KACZYŃSKI \\ Warsaw University of Technology, Faculty of Mathematics and Information Science, Warsaw, Poland \\ e-mail: akacz@mini.pw.edu.pl \\ BOHDAN MONASTYRSKYY \\ Protein Structure Prediction Center, University of California Davis, Davis, CA USA; e-mail: bmonast@gmail.com
}

\begin{abstract}
An analytical solution of a 3D transversely isotropic thermoelastic problem of a uniform heat flow disturbed by a penny-shaped rigid sheet-like inclusion (anticrack) with some small conductivity is obtained by using the potential theory method. The behaviour of thermal stresses near the edge of the disc is analysed from the standpoint of the mechanics of fracture initiation.
\end{abstract}

Keywords: transversely isotropic space, circular anticrack, heat flow, singular integral equations, thermal stress singularities

\section{Introduction}

The study of thermal stresses in solids containing foreign inhomogeneities has great importance for the evaluation of the strength of materials and structures which operate under thermal actions. The rapid development of high-strength composite materials has driven researches to take into account the influence of anisotropy in thermomechanical fields for fractured bodies. In addition to cracks, rigid lamellate inclusions (also called anticracks, for brevity) are objects around which stress concentrations occur, which will stimulate failure of materials. Most of research works discuss $2 \mathrm{D}$ problems dealing with these defects. Owing to mathematical complexity, only few publications on the subject within 3D statement of thermoelastic anticrack problems can be found in the literature (see Kit and Khay, 1989; Stadnyk, 1994, 2011; Podil'chuk, 2001; Chaudhuri, 2003; Kaczyński and Kozłowski, 2009; Kaczyński and Monastyrskyy, 2013).

This work treats a rigid penny-shaped inclusion obstructing a uniform perpendicular heat flow in a transversely isotropic space. It may be regarded as a sequel to our papers (Kaczyński and Monastyrskyy, 2009; Kaczyński, 2014; see also extensive references therein) in which a classical condition of thermal insulation of the inclusion faces was assumed. The present contribution focuses on the determination of a stationary temperature field with more general thermal condition by taking into account certain conductivity of a rigid inclusion. The associated problem of induced thermal stresses is reduced to a two-dimensional singular equation with the unknown normal stress discontinuity across inclusion faces, a closed-form solution to which is found by use of Dyson and Galin theorems. Relations for the evaluation of stresses near the inclusion edge are presented and interpreted from a fracture perspective. Moreover, thermal and mechanical fields for thermally conductive and insulated anticracks are compared.

\section{Thermoelastostatics of transversely isotropic materials}

Let us recall the basic relations of uncoupled thermoelasticity for homogeneous transversely isotropic materials. Referring to a Cartesian coordinate system $\left(X_{1}, X_{2}, X_{3}\right)$ and denoting the 
temperature, fluxes, displacements and stresses by $T, q_{i}, u_{i}, \sigma_{i j}$, respectively, the governing equations for an infinite transversely isotropic thermoelastic solid whose isotropic plane is perpendicular to the $X_{3}$-axis and, in absence of body forces and heat sources, are (Ding et al., 2006; Kaczyński, 2014)

$$
\begin{aligned}
& T_{, \gamma \gamma}+k_{0}^{-2} T_{, 33}=0 \\
& q_{\alpha}=-k_{1} T_{, \alpha} \quad q_{3}=-k_{3} T_{, 3} \\
& \frac{1}{2}\left(c_{11}+c_{12}\right) u_{\gamma, \gamma \alpha}+\frac{1}{2}\left(c_{11}-c_{12}\right) u_{\alpha, \gamma \gamma}+c_{44} u_{\alpha, 33}+\left(c_{13}+c_{44}\right) u_{3,3 \alpha}=\beta_{1} T_{, \alpha} \\
& \left(c_{13}+c_{44}\right) u_{\gamma, \gamma 3}+c_{44} u_{3, \gamma \gamma}+c_{33} u_{3,33}=\beta_{3} T_{, 3} \\
& \sigma_{3 \alpha}=c_{44}\left(u_{\alpha, 3}+u_{3, \alpha}\right) \\
& \sigma_{33}=c_{13} u_{\gamma, \gamma}+c_{33} u_{3,3}-\beta_{3} T \\
& \sigma_{12}=\frac{1}{2}\left(c_{11}-c_{12}\right)\left(u_{1,2}+u_{2,1}\right) \\
& \sigma_{11}=c_{11} u_{1,1}+c_{12} u_{2,2}+c_{13} u_{3,3}-\beta_{1} T \\
& \sigma_{22}=c_{12} u_{1,1}+c_{11} u_{2,2}+c_{13} u_{3,3}-\beta_{1} T
\end{aligned}
$$

Moreover,

$$
k_{0}=\sqrt{\frac{k_{1}}{k_{3}}} \quad \beta_{1}=\left(c_{11}+c_{12}\right) \alpha_{1}+c_{13} \alpha_{3} \quad \beta_{3}=2 c_{13} \alpha_{1}+c_{33} \alpha_{3}
$$

In the equations given above, $k_{1}\left(\alpha_{1}\right)$ and $k_{3}\left(\alpha_{3}\right)$ denote the coefficients of conductivity (of thermal expansion) in the plane isotropy and along the $X_{3}$-axis of rotational material symmetry, respectively, and $c_{11}, c_{12}, c_{13}, c_{33}, c_{44}$ are five independent elastic constants. Indices $i, j$ run over $1,2,3$ while indices $\alpha, \gamma$ run over 1,2 . Summation convention holds unless otherwise stated. Subscripts preceded by a comma indicate partial differentiation with respect to the corresponding coordinates.

\section{Formulation of the anticrack problem}

Consider a transversely isotropic space weakened by a penny-shaped rigid inclusion (anticrack) subjected to a uniform steady-flow of heat $q_{0}$ in the direction of the negative $X_{3}$-axis as shown in Fig. 1. The anticrack region $S$ on the mid-plane of transverse isotropy $x_{3}=0$ is denoted as $r \equiv \sqrt{x_{1}^{2}+x_{2}^{2}} \leqslant a$.

We are faced with the boundary-value value problem: find the fields $T$ and $u_{i}$ suitable smooth on $R^{3}-S$ such that Eqs. (2.1) hold, subject to the following boundary conditions:

- thermal conditions taking into account the thermal conductivity within the anticrack $S$

- mechanical conditions for $\left(x_{1}, x_{2}, x_{3}=0^{ \pm}\right) \in S$ with a small constant $\varepsilon$ characterizing the rigid vertical translation

$$
u_{1}=u_{2}=0 \quad u_{3}=\varepsilon
$$

— thermal and mechanical conditions at infinity

$$
\begin{aligned}
& q_{1}=q_{2}=0 \quad q_{3}=-q_{0} \quad\left(q_{0}>0\right) \\
& \sigma_{i j}=0
\end{aligned}
$$




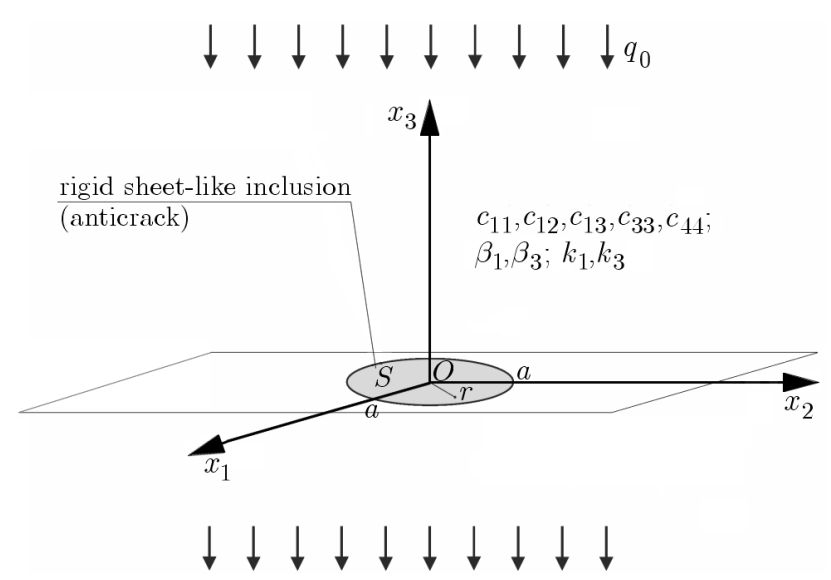

Fig. 1. A transversely isotropic space with a penny-shaped conductive anticrack subjected to a perpendicular uniform flow

\section{Solution}

By means of the superposition principle, it is convenient to represent the solution to the anticrack boundary-value problem as a sum of two components, namely

$$
T=T^{(0)}+\widetilde{T} \quad u_{i}=u_{i}^{(0)}+\widetilde{u}_{i} \quad \sigma_{i j}=\sigma_{i j}^{(0)}+\widetilde{\sigma}_{i j}
$$

where the components attached by 0 describe the basic state of the defect-free solid, and the components with the tilde represent perturbations due to the anticrack.

The results for the first 0-problem are found to be given by Kaczyński (2014)

$$
\begin{aligned}
T^{(0)} & =\frac{q_{0}}{k_{3}} x_{3} \\
u_{\alpha}^{(0)} & =\frac{\beta_{3} q_{0}}{k_{3}\left(2 c_{13}+c_{33}\right)} x_{\alpha} x_{3} \quad u_{3}^{(0)}=\frac{\beta_{3} q_{0}}{2 k_{3}\left(2 c_{13}+c_{33}\right)}\left(x_{3}^{2}-x_{1}^{2}-x_{2}^{2}\right) \\
\sigma_{i j}^{(0)} & =0
\end{aligned}
$$

Attention will be next drawn to the corrective solution of the perturbed problem. The disturbing thermal field $\widetilde{T}$, decaying at infinity, is determined by solving quasi-Laplace equation $(2.1)_{1}$ with applying the following model expressions related to the rigid disc $S$, given from Kaczyński and Monastyrskyy (2009)

$$
\begin{aligned}
& \widetilde{T}_{, 3}\left(x_{1}, x_{2}, x_{3}=0^{+}\right)-\widetilde{T}_{, 3}\left(x_{1}, x_{2}, x_{3}=0^{-}\right)=0 \\
& \widetilde{T}\left(x_{1}, x_{2}, 0^{+}\right)-\widetilde{T}\left(x_{1}, x_{2}, 0^{-}\right)-k_{3} R\left(x_{1}, x_{2}\right) \widetilde{T}_{, 3}\left(x_{1}, x_{2}, 0^{+}\right)=q_{0} R\left(x_{1}, x_{2}\right)
\end{aligned}
$$

where $R\left(x_{1}, x_{2}\right)$ is interpreted as the thermal anticrack resistance.

From the potential theory (Kellogg, 1953), the solution is expressed as follows

$$
\widetilde{T}\left(x_{1}, x_{2}, x_{3}\right)=\left.\frac{\partial \widetilde{\omega}\left(x_{1}, x_{2}, z_{0}\right)}{\partial z_{0}}\right|_{z_{0}=k_{0} x_{3}}
$$

with

$$
\widetilde{\omega}\left(x_{1}, x_{2}, z_{0}\right)=-\frac{1}{2 \pi} \iint_{S} \frac{\omega\left(\xi_{1}, \xi_{2}\right) d \xi_{1} d \xi_{2}}{\sqrt{\left(x_{1}-\xi_{1}\right)^{2}+\left(x_{2}-\xi_{2}\right)^{2}+z_{0}^{2}}}
$$


Using the well-known property of a simple layer potential, the satisfaction of Eq. $(4.3)_{2}$ leads to an integro-differential singular equation of the Newton type for the unknown density of the potential of the single layer $\omega\left(\xi_{1}, \xi_{2}\right)$

$$
2 \omega\left(x_{1}, x_{2}\right)-\frac{\sqrt{k_{1} k_{3}} R\left(x_{1}, x_{2}\right)}{2 \pi} \Delta \iint_{S} \frac{\omega\left(\xi_{1}, \xi_{2}\right) d \xi_{1} d \xi_{2}}{\sqrt{\left(x_{1}-\xi_{1}\right)^{2}+\left(x_{2}-\xi_{2}\right)^{2}}}=q_{0} R\left(x_{1}, x_{2}\right)
$$

in which $\Delta \equiv \frac{\partial^{2}}{\partial x_{1}^{2}}+\frac{\partial^{2}}{\partial x_{2}^{2}}$ stands for the two-dimensional Laplace operator. Assuming next that

$$
R\left(x_{1}, x_{2}\right)=\widetilde{R}(r)=R_{0} \sqrt{a^{2}-r^{2}} \quad R_{0}>0
$$

an analytical solution to Eq. (4.6) is achieved in the form

$$
\omega\left(x_{1}, x_{2}\right)=\widetilde{\omega}(r)=\frac{2 \widetilde{q}}{\pi \sqrt{k_{1} k_{3}}} \sqrt{a^{2}-r^{2}}
$$

with

$$
\widetilde{q}=q_{0}\left(1+\frac{4}{\pi \sqrt{k_{1} k_{2}} R_{0}}\right)^{-1} \leqslant q_{0}
$$

Inserting Eq. (4.8) into (4.5) and after integration we arrive at the following elementary formulas for the main thermal potential $\widetilde{\omega}$ for $x_{3} \geqslant 0$ (see Fabrikant, 1989)

$$
\widetilde{\omega}\left(x_{1}, x_{2}, z_{0}\right)=-\frac{\widetilde{q}}{2 \pi \sqrt{k_{1} k_{3}}}\left[\left(2 a^{2}+2 z_{0}^{2}-r^{2}\right) \sin ^{-1} \frac{a}{l_{20}}-\frac{2 a^{2}-3 l_{10}^{2}}{a} \sqrt{l_{20}^{2}-a^{2}}\right]
$$

and, in view of Eqs. (4.4) and $(2.1)_{2}$, for the temperature $\widetilde{T}$ and heat fluxes $\widetilde{q}_{i}$

$$
\begin{aligned}
& \widetilde{T}\left(x_{1}, x_{2}, x_{3}\right)=-\frac{2 \widetilde{q}}{\pi \sqrt{k_{1} k_{3}}}\left(k_{0} x_{3} \sin ^{-1} \frac{a}{l_{20}}-\sqrt{a^{2}-l_{10}^{2}}\right) \\
& \widetilde{q}_{\alpha}=\frac{2 \widetilde{q} a^{2}}{\pi} \sqrt{\frac{k_{1}}{k_{3}}} \frac{x_{\alpha} \sqrt{a^{2}-l_{10}^{2}}}{l_{20}^{2}\left(l_{20}^{2}-l_{10}^{2}\right)} \quad \widetilde{q}_{3}=\frac{2 \widetilde{q}}{\pi}\left(\sin ^{-1} \frac{a}{l_{20}}-\frac{a \sqrt{l_{20}^{2}-a^{2}}}{l_{20}^{2}-l_{10}^{2}}\right)
\end{aligned}
$$

Here

$$
\begin{array}{ll}
l_{1}=l_{1}\left(x_{3}\right)=\frac{1}{2}\left[\sqrt{(r+a)^{2}+x_{3}^{2}}-\sqrt{(r-a)^{2}+x_{3}^{2}}\right] & l_{10}=l_{1}\left(z_{0}\right) \\
l_{2}=l_{2}\left(x_{3}\right)=\frac{1}{2}\left[\sqrt{(r+a)^{2}+x_{3}^{2}}+\sqrt{(r-a)^{2}+x_{3}^{2}}\right] & l_{20}=l_{2}\left(z_{0}\right)
\end{array}
$$

In the inclusion plane $x_{3}=0^{ \pm}$(making use of the relations $\left.l_{10}\right|_{x_{3}=0}=\min (a, r),\left.l_{20}\right|_{x_{3}=0}=$ $\max (a, r))$, we obtain

$$
\begin{aligned}
& T\left(r, 0^{ \pm}\right)= \begin{cases} \pm \frac{2 \widetilde{q}}{\pi \sqrt{k_{1} k_{3}}} \sqrt{a^{2}-r^{2}} & 0 \leqslant r \leqslant a \\
0 & r>a\end{cases} \\
& q_{r}\left(r, 0^{ \pm}\right)=-k_{1} \frac{\partial T\left(r, 0^{ \pm}\right)}{\partial r}= \begin{cases} \pm \frac{2 \widetilde{q}}{\pi} \sqrt{\frac{k_{1}}{k_{3}}} \frac{r}{\sqrt{a^{2}-r^{2}}} & 0 \leqslant r \leqslant a \\
0 & r>a\end{cases} \\
& q_{3}\left(r, 0^{ \pm}\right)=-k_{3} T_{3}\left(r, 0^{ \pm}\right)= \begin{cases}\widetilde{q}-q_{0} & 0 \leqslant r<a \\
\frac{2 \widetilde{q}}{\pi}\left(\sin ^{-1} \frac{a}{r}-\frac{a}{\sqrt{r^{2}-a^{2}}}\right)-q_{0} & r>a\end{cases}
\end{aligned}
$$


It follows from these formulas that the rigid inclusion acts as an obstruction to the given heat flow, producing thermal local disturbances such as the jump of temperature $T$ across the inclusion plane and the infinite increase of the heat fluxes in the interior vicinity of the inclusion edge.

Now we pass to the non-trivial perturbed elastic problem, affixed by the tilde and connected with the determination of the induced state of stress and deformation resulting from the known disturbed temperature $\widetilde{T}=\left.\left(\partial \widetilde{\omega} / \partial z_{0}\right)\right|_{z_{0}=k_{0} x_{3}}$. Because of the anti-symmetry of the temperature and stress system, and bearing in mind Eqs. (3.1), (4.1) and (4.2), it reduces to that of the half space $x_{3} \geqslant 0$ subjected to the following mixed boundary conditions

$$
\begin{array}{ll}
\widetilde{u}_{\alpha}\left(x_{1}, x_{2}, x_{3}=0^{+}\right)=0 \quad\left(x_{1}, x_{2}\right) \in R^{2} & \\
\widetilde{u}_{3}\left(x_{1}, x_{2}, x_{3}=0^{+}\right)=\frac{\beta_{3} q_{0}}{2 k_{3}\left(2 c_{13}+c_{33}\right)}\left(x_{1}^{2}+x_{2}^{2}\right)+\varepsilon \quad\left(x_{1}, x_{2}\right) \in S
\end{array}
$$

and

$$
\begin{aligned}
& \widetilde{\sigma}_{33}\left(x_{1}, x_{2}, x_{3}=0^{+}\right)=0 \quad\left(x_{1}, x_{2}\right) \in R^{2}-S \\
& \widetilde{u}_{i}=O\left(|x|^{-1}\right) \quad|x|=\sqrt{x_{1}^{2}+x_{2}^{2}+x_{3}^{2}} \rightarrow \infty
\end{aligned}
$$

Moreover, having found the distribution of the normal stress $\left.\widetilde{\sigma}_{33}\right|_{S^{+}} \equiv q\left(x_{1}, x_{2}\right)$ in the region $S$, the unknown rigid translation $\varepsilon$ can be calculated from the equilibrium condition

$$
\iint_{S} q\left(x_{1}, x_{2}\right) d x_{1} d x_{2}=0
$$

A solution to this problem was given by Kaczyński (2014). Here only the main idea and final results with some modifications will be presented.

An efficient approach is based on the construction of harmonic potentials that satisfy governing equations $(2.1)_{3,4}$ and are well suited to the above-mentioned anticrack boundary conditions. We take the following displacement representation expressed by potentials $\widetilde{\phi}_{\alpha} \equiv \widetilde{\phi}_{\alpha}\left(x_{1}, x_{2}, z_{\alpha}\right)$, $z_{\alpha}=t_{\alpha} x_{3}, \alpha=1$ or $\alpha=2$

$$
\widetilde{u}_{\alpha}=\left(\widetilde{\phi}_{1}+\widetilde{\phi}_{2}+c_{1} \int_{z_{0}}^{\infty} \widetilde{\omega}\left(x_{1}, x_{2}, z_{0}\right) d z_{0}\right)_{, \alpha} \quad \widetilde{u}_{3}=m_{\alpha} t_{\alpha} \frac{\partial \widetilde{\phi}_{\alpha}}{\partial z_{\alpha}}+c_{2} k_{0} \widetilde{\omega}
$$

with the potentials satisfying the harmonic equations

$$
\left(\Delta+\frac{\partial^{2}}{\partial z_{\alpha}^{2}}\right) \widetilde{\phi}_{\alpha}=0 \quad \alpha=1,2 \quad(\text { no sum on } \alpha)
$$

Here the constants $m_{\alpha}, c_{\alpha}, t_{\alpha}$ are given in Appendix A of Kaczyński (2014). Note that the general case $t_{1} \neq t_{2}, t_{\alpha} \neq k_{0}$ is considered.

Next we put

$$
\widetilde{\phi}_{\alpha}=(-1)^{\alpha} \widetilde{f}\left(x_{1}, x_{2}, z_{\alpha}\right)+a_{\alpha} \int_{z_{\alpha}}^{\infty} \widetilde{\omega}\left(x_{1}, x_{2}, z_{\alpha}\right) d z_{\alpha} \quad \alpha=1,2 \quad(\text { no } \operatorname{sum} \text { on } \alpha)
$$

where

$$
\left(\Delta+\frac{\partial}{\partial x_{3}^{2}}\right) \widetilde{f}\left(x_{1}, x_{2}, x_{3}\right)=0
$$


and choose the constants $a_{\alpha}$ in order to satisfy a part of boundary conditions (3.1). In this way, the perturbed anticrack problem reduces to the determination of a potential function $\tilde{f}$ in the upper half-space, decaying at infinity and satisfying the following mixed conditions on the $X_{1} X_{2}$-plane

$$
\begin{aligned}
& \left.\frac{\partial \widetilde{f}\left(x_{1}, x_{2}, x_{3}\right)}{\partial x_{3}}\right|_{x_{3}=0^{+}}=\frac{1}{m_{2} t_{2}-m_{1} t_{1}} f_{0}\left(x_{1}, x_{2}\right) \quad\left(x_{1}, x_{2}\right) \in S \\
& \left.\frac{\partial^{2} \widetilde{f}\left(x_{1}, x_{2}, x_{3}\right)}{\partial x_{3}^{2}}\right|_{x_{3}=0^{+}}=0 \quad\left(x_{1}, x_{2}\right) \in R^{2}-S
\end{aligned}
$$

where

$$
f_{0}\left(x_{1}, x_{2}\right)=\widetilde{f}_{0}(r)=-\beta^{*} \widetilde{\omega}(r, 0)+A r^{2}+\varepsilon=\frac{\beta^{*} \widetilde{q} a^{2}}{2 \sqrt{k_{1} k_{3}}}+\varepsilon+\left(A-\frac{\beta^{*} \widetilde{q}}{4 \sqrt{k_{1} k_{3}}}\right) r^{2}
$$

with the following constants

$$
\begin{array}{lcc}
\beta^{*}=c_{2} k_{0}-a_{\alpha} m_{\alpha} t_{\alpha} & a_{1}=\frac{c_{1}\left(1+m_{2}\right)-\frac{\delta_{3}}{c_{44}}}{m_{1}-m_{2}} & a_{2}=\frac{-c_{1}\left(1+m_{1}\right)+\frac{\delta_{3}}{c_{44}}}{m_{1}-m_{2}} \\
\delta_{3}=\beta_{3}-c_{1} c_{13}-c_{2} c_{33} k_{0}^{2} & A=\frac{\beta_{3} q_{0}}{2 k_{3}\left(2 c_{13}+c_{33}\right)} &
\end{array}
$$

It is known from the potential theory (Kellogg, 1953) that the solution to this problem is represented by the Newton potential of a simple layer distributed over the region $S$ as

$$
\widetilde{f}\left(x_{1}, x_{2}, x_{3}\right)=\frac{1}{2 \pi c_{44}\left(m_{1}-m_{2}\right)} \iint_{S} q\left(\xi_{1}, \xi_{2}\right) \ln \left(\sqrt{\left(x_{1}-\xi_{1}\right)^{2}+\left(x_{2}-\xi_{2}\right)^{2}+x_{3}^{2}}+x_{3}\right) d \xi_{1} d \xi_{2}
$$

where the unknown layer density $q$ can be identified as the normal stress $\left.\widetilde{\sigma}_{33}\right|_{S^{+}}$. Taking consideration of the first condition in Eq. (4.21), the following governing two-dimensional singular integral equation (similar to that arising in classical contact mechanics) is obtained

$$
\widetilde{H} \iint_{S} \frac{q\left(\xi_{1}, \xi_{2}\right) d \xi_{1} d \xi_{2}}{\sqrt{\left(x_{1}-\xi_{1}\right)^{2}+\left(x_{2}-\xi_{2}\right)^{2}}}=-f_{0}\left(x_{1}, x_{2}\right) \quad\left(x_{1}, x_{2}\right) \in S
$$

with $f_{0}$ given by Eq. (4.22) and $\widetilde{H}$ defined by

$$
\widetilde{H}=\frac{m_{2} t_{2}-m_{1} t_{1}}{2 \pi c_{44}\left(m_{2}-m_{1}\right)}=\frac{\sqrt{c_{11} c_{33}}+c_{44}}{2 \pi \sqrt{c_{44} c_{33}} \sqrt{\left(\sqrt{c_{11} c_{33}}-c_{13}\right)\left(\sqrt{c_{11} c_{33}}+c_{13}+2 c_{44}\right)}}
$$

Taking a solution to this equation in the form (using Dyson's and Galin's theorems)

$$
q\left(x_{1}, x_{2}\right)=\widetilde{q}(r)=\frac{\widetilde{p}_{0} a^{2}-\widetilde{p}_{2} r^{2}}{\widetilde{H} \pi^{2} \sqrt{a^{2}-r^{2}}} \quad 0 \leqslant r<a
$$

and substituting it into Eq. (4.25), after appropriate calculations and utilizing Eq. (4.16), we find the unknown coefficients $\widetilde{p}_{0}$ and $\widetilde{p}_{2}$ as well as the rigid vertical displacement $\varepsilon$

$$
\widetilde{p}_{2}=4 A-\frac{\beta^{*} \widetilde{q}}{\sqrt{k_{1} k_{3}}} \quad \widetilde{p}_{0}=\frac{2}{3} \widetilde{p}_{2} \quad \varepsilon=-a^{2}\left(2 A-\frac{1}{3} \widetilde{p}_{2}\right)
$$


The primary harmonic potential to the thermoelastic perturbed problem is obtained by calculating integral (4.24) with the use of Eq. (4.27). From the results given in Fabrikant (1989, 1991), it is found that for $x_{3} \geqslant 0$

$$
\begin{gathered}
\tilde{f}\left(r, x_{3}\right)=-\frac{\widetilde{p}_{2}}{3 \pi^{2}\left(m_{2} t_{2}-m_{1} t_{1}\right)}\left[x_{3} \sin ^{-1} \frac{a}{l_{2}}\left(a^{2}-\frac{3}{2} r^{2}+x_{3}^{2}\right)\right. \\
\left.+\sqrt{a^{2}-l_{1}^{2}}\left(\frac{5}{2} r^{2}+\frac{1}{3} a^{2}-l_{2}^{2}-\frac{11}{6} l_{1}^{2}\right)\right]
\end{gathered}
$$

The expressions for the full-space stress-displacement field can then be obtained from Eq. (4.29) by simple differentiation, with all results being in terms of elementary functions. As easily seen, the solution is axially symmetric. In particular, let us focus on some quantities in the anticrack plane which are presented below

$$
\begin{aligned}
& u_{1}\left(r, 0^{ \pm}\right)=u_{2}\left(r, 0^{ \pm}\right)=0 \quad 0 \leqslant r<\infty \\
& u_{3}\left(r, 0^{ \pm}\right)= \begin{cases}\varepsilon & 0 \leqslant r<a \\
\frac{2}{\pi}\left(\varepsilon+A r^{2}\right) \sin ^{-1} \frac{a}{r}-\frac{2 A a}{\pi} \sqrt{r^{2}-a^{2}}-A r^{2} & r>a\end{cases} \\
& \sigma_{33}\left(r, 0^{ \pm}\right)= \begin{cases} \pm \frac{\widetilde{p}_{2}}{3 \widetilde{H} \pi^{2}} \frac{2 a^{2}-3 r^{2}}{\sqrt{a^{2}-r^{2}}} & 0 \leqslant r<a \\
0 & r>a\end{cases} \\
& \sigma_{3 r}\left(r, 0^{ \pm}\right)= \begin{cases}\widetilde{\beta} r & 0 \leqslant r<a \\
\frac{2}{\pi}\left(\widetilde{\beta} r \sin ^{-1} \frac{a}{r}-\frac{\widetilde{\beta}_{0} a^{3}}{r \sqrt{r^{2}-a^{2}}}-\frac{\left.\widetilde{\beta} a \sqrt{r^{2}-a^{2}}\right)}{r}\right) & r>a\end{cases}
\end{aligned}
$$

where

$$
\begin{aligned}
& \widetilde{\beta}_{0}=\widetilde{p}_{2} \frac{c_{44}\left(\sqrt{c_{11} c_{33}}-c_{13}\right)}{3\left(\sqrt{c_{11} c_{33}}+c_{44}\right)} \quad \widetilde{\beta}=\frac{c_{44}}{2}\left[3 \widetilde{\beta}_{0}-q_{0} \frac{\widetilde{\delta}+\left(c_{1}-c_{2}\right) k_{0}}{\sqrt{k_{1} k_{2}}}\right] \\
& \widetilde{\delta}=\frac{\left(\sqrt{c_{11} c_{33}}-c_{13}\right)\left(2 c_{1} c_{44}-\delta_{3}\right)}{c_{33} c_{44}\left(t_{1}+t_{2}\right)}-c_{1}\left(t_{1}+t_{2}\right)
\end{aligned}
$$

\section{Analysis of the results and conclusions}

The analytical results obtained in the previous Section are useful in interpreting the mechanics of fracture initiation at the rim of the rigid inclusion. In view of linear fracture mechanics, two failure mechanisms are possible: mode II (edge-sliding) of fracture deformation characterized by the stress intensity factor

$$
K_{\mathrm{II}}=\lim _{r \rightarrow a^{+}} \sqrt{2 \pi(r-a)} \sigma_{3 r}(r, 0)=-\frac{2 \widetilde{\beta}_{0} a \sqrt{a}}{\sqrt{\pi}}
$$

and the possible detachment of the material from the inclusion surface described by the stress intensity coefficients

$$
S_{\mathrm{I}}^{ \pm}=\lim _{r \rightarrow a^{-}} \sqrt{2 \pi(a-r)} \sigma_{33}\left(r, 0^{ \pm}\right)=\mp \frac{\widetilde{p}_{2} a \sqrt{a}}{3 \pi \sqrt{\pi} \widetilde{H}}
$$

These parameters can be used in conjunction with a suitable failure criterion.

In conclusion, by taking into account some interior conductivity of the anticrack, we have pointed out that by letting $R_{0} \rightarrow \infty$ (see Eqs. (4.6) and (4.7)) the present solution with 
$\widetilde{q}=q_{0}$ (cf. Eq. (4.9)) reduces to that dealing with the case of a thermally insulated rigid circular inclusion obtained in Kaczyński (2014). Moreover, comparison between thermally conductive and insulated anticracks in a transversely isotropic (in particular, isotropic) space has shown only quantitative changes in the temperature and stress distributions.

\section{References}

1. Chaudhuri R.A., 2003, Three-dimensional asymptotic stress field in the vicinity of the circumference of a penny-shaped discontinuity, International Journal of Solids and Structures, 40, 3787-3805

2. Ding H., Chen W., Zhang L., 2006, Elasticity of Transversely Isotropic Materials, Solid Mechanics and its Applications, 126, Springer, The Netherlands

3. Fabrikant V.I., 1989, Applications of Potential Theory in Mechanics: A Selection of New Results, Kluver Academic Publishers, Dordrecht

4. Fabrikant V.I., 1991, Mixed Boundary Value problems of Potential Theory and their Applications, Kluver Academic Publishers, Dordrecht

5. KACZYŃSKi A., 2014, Thermal stress analysis of a three-dimensional anticrack in a transversely isotropic solid, International Journal of Solids and Structures, 51, 2382-2389

6. KaCZYŃSKi A., KozŁowski W., 2009, Thermal stresses in an elastic space with a perfectly rigid flat inclusion under perpenicular heat flow, International Journal of Solids and Structures, 46, $1772-1777$

7. KaCZYŃski A., Monastyrskyy B., 2009, On the thermoelastic problem of uniform heat flow disturbed by a circular rigid lamellate inclusion, Archives of Mechanics, 61, 3/4, 309-324

8. KaczyŃski A., Monastyrskyy B., 2013, A rigid inclusionin an elastic space under the action of a uniform heat flow in the inclusion plane, International Journal of Solids and Structures, 50, 2631-2640

9. Kellogg O.D., 1953, Foundation of Potential Theory, Dover, New York

10. Kit H.S, Khay M.V., 1989, Method of Potentials in Three-Dimensional Thermoelastic Problems of Bodies with Cracks (in Russian), Naukova Dumka, Kiev

11. Podil'Chuk Yu.,N., 2001, Exact analytical solutions of three-dimensional static thermoelastic problems for a transversally isotropic body in curvilinear coordinate systems, International Applied Mechanics, 37, 6, 728-761

12. Stadnyk M.M., 1994, Method for the solution of the three-dimensional thermoelastcity for bodies with thin inclusions, Materials Science, 30, 6, 643-653

13. StadnYK M.M., 2011, Elastic-ellipsoidal heat-conducting inclusion in a body under the action of a heat flux applied at infinity, Materials Science, 47, 3, 284-293 